\title{
EL JUEGO EN LA LICENCIATURA EN EDUCACIÓN FÍSICA DE LA UNIVERSIDAD PEDAGÓGICA NACIONAL, DESDE LA PERSPECTIVA DE PROFESORES ${ }^{1}$
}

\author{
GAMES IN THE PHYSICAL EDUCATION PROGRAM OF UNIVERSIDAD PEDAGÓGICA NACIONAL \\ FROM THE PERSPECTIVE OF TEACHERS
}

\section{O JOGO NA LICENCIATURA EM EDUCAÇÃO FÍSICA DA UNIVERSIDADE PEDAGÓGICA NACIONAL DESDE A PERSPECTIVA DE PROFESSORES}

José Orlando Pachón Moreno ${ }^{2}$

\section{Resumen}

\begin{abstract}
Desde la vinculación del programa Educación Física a la Universidad Pedagógica Nacional en el año 1962 hasta la actualidad, se reconocen tres planes curriculares en la formación de los licenciados de esta área educativa dentro de los cuales el juego ha estado presente principalmente desde la perspectiva didáctica, esto es, como mediación en los procesos de enseñanza/aprendizaje. Su estudio ha llevado incluso a proponerlo como el sustento de una posible alternativa de educación física, suscitando el debate entre ser medio y ser fin en los procesos educativos. El interés de este artículo se centra en compendiar, desde la óptica de profesores de las diferentes propuestas curriculares, algunos de ellos como previos estudiantes, las diversas teorías y prácticas que se han desarrollado acerca del juego en los tres planes curriculares. Se realizaron entrevistas semi-estructuradas a diez (10) profesores de la Facultad de Educación Física de la Universidad en el marco del proyecto de Doctorado en Educación "El Juego como objeto de enseñanza en la Educación Física" en el primer semestre académico de 2016. Como resultado del estudio, además de la pretensión de establecer múltiples líneas de reflexión que permitan fortalecer y dar continuidad a la argumentación del juego en el ámbito de la educación física, se aboga por continuar con la construcción del conocimiento profesional de sus profesores.
\end{abstract}

Palabras clave: planes curriculares; juego; educación física; mediación; fin

1 En el marco del proyecto de Doctorado en Educación de la UPN, "El Juego como objeto de enseñanza en la Educación Física" (desarrollado por el autor del artículo), en el primer semestre académico de 2016 se estableció diálogo (existe registro en audio) sobre la presencia del juego en los diferentes planes curriculares para la Licenciatura en Educación Física en la Universidad Pedagógica Nacional con profesores, quienes serán referidos a través de abreviaturas a lo largo del artículo; Los datos de cada docente se incluyen al final.

2 Doctorando, Doctorado Interinstitucional en Educación (UPN), Máster en Didáctica Contemporánea de la Educación Física, La Habana (Cuba), Magíster en Desarrollo Educativo y Social Cinde-UPN, Licenciado en Educación Física UPN. Profesor de planta de la UPN, Facultad de Educación Física. Correo electrónico: jopachon@pedagogica.edu.co orlandopachon@gmail.com 


\section{Abstract}

From creation of the Physical Education Program at Universidad Pedagógica Nacional in 1962 to this day, there have been three curricular plans related to the training of Physical Education teachers, where games have been present mainly from the didactic perspective, that is, as mediation in the teaching/learning processes. Its study has even led to proposing games as the basis of a possible alternative to physical education, sparking the debate between being the means and being the end in the educational processes. The main purpose of this article is to compile the various theories and practices developed about games in the three curricular plans from the point of view of teachers from the different curricular proposals (some of them as previous students). Semi-structured interviews were conducted with ten (10) professors from the university's School of Physical Education in the context of the PhD dissertation titled "Games as Object of Teaching in Physical Education" in the first academic semester of 2016. As a result of the study, and in addition to the intention to establish multiple lines of reflection to strengthen and give continuity to the argumentation of the game in the context of physical education, we advocated for continuing to build on the professional knowledge of P.E. teachers.

Keywords: curricular plans; games; physical education; mediation; end

\section{Resumo}

Desde a vinculação do programa Educação Física à Universidade Pedagógica Nacional no ano 1962 até hoje, são reconhecidos três planos curriculares na formação dos licenciados desta área educativa dentro dos que o jogo está presente, principalmente desde a perspectiva didática, ou seja, como mediação nos processos de ensino/aprendizagem. Seu estudo levou a propô-lo como o sustento de uma possível alternativa da educação física, referenciando o debate entre ser meio e ser fim nos processos educativos. 0 interesse deste artigo é compendiar, desde o ponto de vista dos professores das diversas propostas curriculares, alguns deles anteriormente estudantes, as diversas teorias e práticas desenvolvidas acerca do jogo nos três planos curriculares. Realizaram-se entrevistas semiestruturadas a dez (10) professores da Faculdade de Educação Física da Universidade no marco do projeto de Doutorado em Educação "O jogo como objeto de ensino na Educação Física" no primeiro semestre académico de 2016. Como resultado do estudo, além da pretensão de estabelecer múltiplas linhas de reflexão que permitam fortalecer e continuar a argumentação do jogo no âmbito da educação física, procura-se continuar com a construção do conhecimento profissional dos seus professores.

Palavras chave: planos curriculares; jogo; educação física; mediação; fim

Fecha de recepción: 29 de julio de 2016

Fecha de aprobación: 17 de diciembre de 2016

Para citar este artículo:

Pachón, J. (2017). El juego en la Licenciatura en Educación Física de la Universidad Pedagógica Nacional desde la perspectiva de profesores. Lúdica Pedagógica, (25), 105-116. 


\section{PRESENTACIÓN}

"El establecimiento denominado Escuela Nacional de Educación Física, sería una de las dependencias de la Universidad Pedagógica Nacional", consagra en su artículo 5 el Decreto 2188 de 1962 (Vaca, 1998, p. 71). A partir de esta fecha es posible sugerir, de manera general, tres planes curriculares en la formación de profesionales de la educación física en la Universidad Pedagógica Nacional (UPN): a) el primero, con ajustes en los años iniciales, se desarrolló desde la fecha del decreto en mención y hasta comienzos de la década de 1980; b) el segundo, iniciado en los años ochenta y hasta el año 2000, conocido en el ámbito de la universidad como el plan del ochenta y cuatro; y c) el tercero, con vida académica desde el año 2000 y vigente en la actualidad, denominado Proyecto Curricular de Licenciatura en Educación Física (PC-LEF).

La presencia del juego en el desarrollo de diferentes asignaturas en los tres currículos ha sido una constante, tanto como actividad para enseñanza/aprendizaje, como también recreativa. Así mismo, su estudio y reflexión teórica vienen teniendo desarrollos importantes desde la perspectiva de didácticas instructivas como mediación para la formación, hasta pensarlo como constitutivo de lo humano y, por tanto, considerarlo como fin en los procesos educativos, lo cual ha exigido argumentación desde la antropología, la filosofía y la pedagogía, principalmente.

Este primer acercamiento a la historia del juego en la formación de Licenciados en Educación Física en la UPN, realizado en diálogo con profesores del programa, aspira a dilucidar el recorrido que ha tenido en algunas de las prácticas educativas a lo largo de los tres currículos, así como los desarrollos conceptuales que vienen permitiendo sustentarlo en la formación de profesionales del área.

\section{EL PRIMER PLAN CURRICULAR}

El plan de estudio del primer currículo incluía la asignatura denominada "Juegos Pedagógicos", recuerda JJ1:

Cuando yo dicté la clase, lo que yo abordaba era teorías del juego para encontrar las bases epistemológicas del juego, entonces teoría del atavismo, la teoría de la preparación para los roles, eran como cinco teorías. Se abordaban las teorías y después se abordaba la clasificación de los juegos, pero desde la perspectiva didáctica. Entonces, por ejemplo juegos de coordinación, juegos de equilibrio, juegos según las capacidades motrices y la meta que yo me ponía era que hiciéramos un fichero de cien juegos, donde se daba el nombre del juego, la descripción del juego, el objetivo del juego, ya. Pero fue una tendencia eminentemente didactizada del juego.

Otras opciones de clasificación se daban desde el escenario posible de su realización en la clase de educación física: como juegos de salón o aula de clase y juegos en el patio o espacio exterior. Según el número de participantes también se podían clasificar en juegos individuales y juegos colectivos, así como de acuerdo con los materiales empleados, por ejemplo juegos con pelotas, juegos con sogas, y también por la forma de su organización. Otra categoría de organización era la denominada juegos populares, la cual incluía aquellos de las tradiciones culturales para la preservación de valores autóctonos, como también para la enseñanza de contenidos temáticos del área.

Desde la tipología de juego denominada formas jugadas, JJ1 explica la relación que en su momento establecía con las etapas de aprendizaje motor:

Las formas jugadas aparecen cuando uno empieza a hablar de teorías de aprendizaje motor. Primero daba teorías de aprendizajes y las teorías de aprendizaje en general tenían tres raíces: el desarrollo, la experiencia y la interacción. Desde el desarrollo yo derivaba todo lo que eran teorías estructuralistas, desde la experiencia todo lo que eran teorías conductistas y desde la interacción todo lo que eran las teorías histórico-sociales. Y entonces nombraba autores relevantes o sobresalientes como Piaget, Skiner y Vygotsky, porque ahí comienzan a aparecer una serie de matices de manejo de las teorías de aprendizaje. Una vez hecha esa visibilización de teorías de aprendizaje, entonces yo hablaba de aprendizaje motor y lo explicaba desde la psicología, la pedagogía y la didáctica y desde la, eh, neurofisiología. A mí el que más me aportó para explicar lo de la neurofisiología fue, el de la didáctica del movimiento, Meinel.

En torno al aprendizaje motor (Meinel, 1977) explica JJ1, también abordaba conceptos de la psicología, la pedagogía y la didáctica, de manera coherente con las tres fases por las cuales transita aquel. La primera fase de aprendizaje motor consiste en la irradiación nerviosa 
central a partir de diversos estímulos motores que desde la psicología constituyen las sensaciones y percepciones, en la pedagogía la ambientación y para la didáctica la asimilación. La segunda etapa de aprendizaje motor o concentración, para la psicología comprende las percepciones y representaciones, desde la pedagogía la fijación y a la didáctica la acomodación. Y la tercera etapa de aprendizaje motor o dominio, desde la psicología comprende la simbolización, en la pedagogía el manejo y para la didáctica la aplicación. A manera de ejemplo, JJ1 expresa, "para la irradiación son adecuadas las formas jugadas y los juegos tradicionales, mientras que para la aplicación o dominio, en la cual se enfatiza lo competitivo, son oportunos los juegos pre-deportivos".

Además de las clasificaciones anteriores, FU2 quien asumió con posterioridad JJ1 la orientación de la asignatura "Juegos Pedagógicos", vincula algunas otras como juegos para la detección de características de personalidad, tal es el caso de condiciones de liderazgo, de empatía; como también juegos para la cohesión de grupos, juegos para el desarrollo de procesos organizativos, entre las que recuerda.

Como parte de su estudio del juego para la formación de los licenciados del área en esa época, a partir del sonido de algunos términos, más que de su grafía, FU2 establece relación entre ellos para proponer una perspectiva de juego, más allá de las prácticas que la cultura ha desarrollado y las cuales refiere con el plural los juegos.

La relación la instaura desde el término iocus procedente del latín y sus significados de broma, gracia, ligereza, entre otros y del cual proviene el vocablo juego (Paredes, 2003), con el término joke del anglosajón que también significa "broma" o "burla", y el término yug del sánscrito "que significa unión, conexión, unificación" (De la Ferriére, s.f.), del cual se origina el vocablo yoga.

Lo central de la reflexión de FU2 se da a partir del yug, desde el cual se comprende el yoga como "la unión del ser individual con el ser universal" (De la Ferriére, s.f., p. 27); así se reconoce la unidad compleja de lo humano integrado en el cosmos y la materia física, según el planteamiento actual de Morin (1999). Esta interpretación de lo humano, más allá de las necesidades y exigencias de la cotidianidad, es posible desde el sentido de juego, en la medida que se constituye en puerta de ingreso al mundo de la vacuidad, de lo improductivo según plantea Caillois (1967), siendo la broma y la gracia unas de sus características.

\section{Advierte FU2:}

Es necesario recordar que se está reflexionando el juego en el contexto de la educación física y por tanto es pertinente referirse a ella, entendiéndola como la comprensión de la condición física de lo humano, para entrar en armonía con la vida, con el universo, concibiéndose como parte misma de ese universo; esto a partir de asumir la física como la ciencia que se ocupa del estudio de los fenómenos universales y obviamente entre estos lo humano.

Se supera, por tanto, el tradicional imaginario de educación física que privilegia la formación en técnicas de movimiento para la competitividad y la búsqueda del triunfo como única finalidad, en un escenario de jerarquías, territorialización, agresividad, codicia y deseos posesivos; aspectos que exigen moverse en los denominados tiempos pasados y futuros, en desconocimiento del presente como única posibilidad de la existencia.

La relación juego/educación física, de manera metafórica, FU2, recordando a Cortázar, la plantea como el "proyecto de convertir el juego en la lengua franca de la naturaleza humana, revelada desde la gratuidad del juego, despropósito sin propósito" (Ortega, s.f.). El juego que se aparta del llamado tiempo real y de lo productivo, "rehúsa ser moneda corriente en la manipulación de los bienes", sigue planteando FU2 basándose en Cortázar (Ortega, s.f., p. 34).

Desde estas perspectivas, el juego en la educación física supera la intención instrumental con que se presenta en las respectivas clases, centrado en el perfeccionamiento de técnicas motoras puestas al servicio de lo eminentemente competitivo en pos del triunfo, del trofeo, del premio, como productos externos al juego mismo, en un ambiente de ganadores y perdedores. Es por tanto la gratuidad condición fundamental del juego, en el cual la acción se da por la acción misma sin esperar frutos externos a ella. En ello, recuerda FU2, se coincide con la condición del juego de ser separado (Caillois, 1967), de no ser la vida corriente o cotidiana, sino, todo lo contrario, escabullirse de ella hacia una esfera transitoria, o mundo emancipado de lo habitual (Huizinga, 1968). 
A partir de este desarrollo argumentativo, y específicamente en el campo de los juegos denominados agon (Caillois, 1967), ratifica FU2, es obvio que el otro con quien se comparte ese momento no es un enemigo y de ahí el sentido del saludo en las artes marciales como respeto, reconocimiento y agradecimiento por compartir ese encuentro o si se quiere extraña danza, muy lejana de ser una vulgar pelea. El contrario no es un enemigo, es un compañero necesario para que el juego resulte, en la construcción conjunta de la obra lúdica, dice FU2 recordando a Gruppe (1976).

En las asignaturas orientadas a la formación en técnicas deportivas, se daba otro tipo de clasificación la cual incluía: juegos predeportivos, juegos de iniciación deportiva, juegos atléticos, juegos denominados mini, como minibaloncesto, y el juego deportivo convencional. Clara diferenciación entre algunos de estos no se presentaba y quizás los predeportivos y los juegos de iniciación se referían a lo mismo. Los denominados mini permitían la realización de festivales de formas de deportes ajustadas a las posibilidades y condiciones de la primera escolaridad, sin las exigencias técnicas ni reglamentarias del deporte convencional. Esta categoría de juegos para la educación física también permitía la propuesta de variantes por parte de los estudiantes a partir de plantearles otros objetivos. Al respecto, trae a la memoria CP3 el diseño de juegos para el manejo del control respiratorio en natación, en los cuales se intentaba mantener en el aire plumones soplándolos o hacer goles con pimpones impulsados por soplos de respiración.

En los últimos años de este primer plan curricular y desde lo metodológico, afirma JJ1, la agrupación de estilos de enseñanza propuestos por Mosston (1978) en estilos de reproducción y estilos de producción, posibilitó que los juegos de cualquiera de las clasificaciones anteriores se utilizaran de manera coherente con los propósitos de aquellas. Tan solo por presentar algunos pocos ejemplos, en los estilos de reproducción en los cuales el interés del aprendizaje se centra en la atención al cómo de las actividades, por esta particularidad metodológica los tipos de juego coherentes serían las formas jugadas y los juegos tradicionales.

Dentro de los estilos de producción, los juegos predeportivos, los juegos de iniciación deportiva o los minijuegos, y obviamente el juego deportivo convencional, que enfatizan el resultado final con primacía de lo competitivo, se consideraban los más coherentes en la línea de lo didáctico.

La asignatura denominada "Campamento", recuerda VCh4, también vinculaba el juego a lo largo de su desarrollo como preparación de las actividades que se llevarían a cabo en la realización del mismo para el cierre del semestre académico. En esta los juegos tenían relación directa con las actividades de campamento, entre otras: construcciones, de orientación, de elaboración y degustación de comidas, de alistado de la leña para la cocina. También en la velada cultural se desarrollaban diferentes juegos no ya competitivos como los anteriores, sino principalmente de diversión e interacción, así como juegos del lenguaje y cuentería. Como diría VCh4: "Hasta la guardia nocturna desarrollada para cuidar el campamento, tendía a convertirse en juego de picardías dado el carácter lúdico que caracteriza a los edufísicos ${ }^{3 " .}$

Así mismo, rememora MR5, en el curso denominado "Rondas" el juego tenía cabida de manera musicalizada en las rondas jugadas del tipo Lobo, ¿dónde estás?, en las cuales se combinaban rimas, canto, acción motriz, en situaciones de juego. Aquí también se elaboraban fichas de rondas en estructura similar a las de juegos pedagógicos. De igual manera, recuerda VCh4, en curso intersemestral de gimnasia aplicada orientada por el profesor Peralta ${ }^{4}$, en el cual se tomaron elementos del deporte para convertirlos en movimientos gimnásticos, muchas de estas actividades en su desarrollo adquirieron sentido de juego.

MR5 conocedora de la escuela alemana de deportes y educación física, refiere cómo los juegos constituyen un elemento fundamental en aquella, y dada su influencia en la educación física en la UPN, es comprensible su presencia en diferentes asignaturas y momentos del primer plan de estudios de la licenciatura. Lo confirman los documentos publicados por Coldeportes (1974) bajo la dirección de la Misión Pedagógica Alemana, para la educación física en la escuela primaria, en los cuales el juego tiene amplia presencia en diferentes modalidades, con sus respectivas orientaciones didácticas. Estos documentos fueron dados en su momento y de manera

3 Término con que, en el lenguaje coloquial, se designa a los licenciados en educación física.

4 Para la época, profesor de las asignaturas gimnásticas, entre otras. Hoy se encuentra jubilado. 
gratuita a los estudiantes de la licenciatura, constituyéndose en recurso bibliográfico importante para el ejercicio profesional. Debido a ello adquiere importante presencia Seybold (1976) con sus trabajos escritos, especialmente el titulado Principios Didácticos para la Educación Física en el cual desarrolla un capítulo sobre el juego presentando orientaciones didácticas para su instalación en las respectivas clases.

\section{Recuerda VCh4:}

Los estudiantes de educación física del primer plan curricular podíamos también cursar la asignatura "Juegos Pedagógicos" en el programa de Preescolar. Allí se daba una orientación específica hacia el desarrollo evolutivo de la infancia y no tanto hacia lo formativo. La realización del curso obedecía a un proceso similar que en educación física, basado en la elaboración de un número considerable de fichas de juegos, las cuales tenían más o menos la misma estructura: nombre del juego, objetivo, descripción, materiales necesarios, relación con la edad del niño, pero este aspecto sí no se incluía en el curso en educación física.

A demás de las asignaturas de educación física en las cuales se estudiaba el juego, por esas épocas, refiere D06:

Otra experiencia fueron los cursos de la FIEP, de la Federación Internacional de Educación Física. En esos cursos conocimos gente del exterior y el juego también era considerado como un elemento teórico, por ejemplo Jhon Andrius ${ }^{5}$, recuerdo, planteaba el juego como una parte de la educación física. Nicolas Moletnicer que era profesor de una Universidad en Texas y enseñaba como educación física escolar habló del juego. Yo recuerdo un poco esos dos personajes de los cursos de la FIEP acerca del juego. Aquino y Zapata que vinieron en un curso de la FIEP a enseñar la psicomotricidad, también mencionaron un poco el juego.

De todas maneras, el acercamiento al juego se dio principalmente en la práctica, pero la reflexión teórica y las revisiones bibliográficas sobre este tuvieron menor énfasis en aquellas épocas, recuerda MR5.

5 Los nombres se han transcrito directamente del audio, según se escucha su pronunciación.

\section{EL SEGUNDO PLAN CURRICULAR O CURRÍCULO DE 1984}

Hacia los inicios de la década de 1980, refiere D06, la Universidad Pedagógica Nacional (UPN) propone renovación de currículo, a la cual deben acogerse los diferentes programas de licenciaturas. A partir de la crítica social al deporte y el cuestionamiento que se venía dando a la tecnificación en prácticas deportivas que se enfatizaba en la Licenciatura de Educación Física hasta ese momento, para la nueva propuesta curricular se opta por nominar las asignaturas de formación deportiva como teoría y método de... (a continuación el nombre del deporte, por ejemplo, teoría y método del baloncesto).

Trae a la memoria Daniel el cuestionamiento que surgió en aquel momento:

Y entonces el juego ¿qué hacemos con el juego? fue la pregunta, porque la perspectiva de Cagigal (1979) en el objeto de estudio fue un poco lo que promulgábamos en el cambio. El objeto de estudio de la educación física va desde el hombre en movimiento y el movimiento del hombre hasta el juego y el deporte, pasando por todo lo que la formación corporal encierra. ¿Sí ve?, y entonces había cinco elementos ahí: el movimiento del hombre, el hombre en movimiento que era un poco la perspectiva biomecánica, el análisis del movimiento, el juego que aparece explícitamente y el deporte en su cercanía.

El juego, entonces, tendría cabida en estas nuevas asignaturas las cuales lo asumirán estableciendo relación entre jugar y hacer deporte, vinculando en su desarrollo los juegos educativos, los juegos predeportivos y el deporte; quedando en manos del docente la reflexión sobre la didáctica desde la cual instalar el juego en el desarrollo de las respectivas clases.

Desde estas perspectivas, ya no se consideró necesaria para el nuevo currículo la presencia de la asignatura "Juegos Pedagógicos", dado que sus contenidos ahora los asumirían las teorías y métodos de los diferentes deportes. La intencionalidad formativa hacía tránsito de la competitividad deportiva, hacia la formación a través del deporte, para la cual el juego se consideraba relevante.

Asumir el juego como medio pedagógico para la educación y el aprendizaje, argumenta D06, limitó la reflexión a ese nivel sin dar apertura al desarrollo de teoría sobre el juego. En este sentido, para el momento fueron 
suficientes algunas ideas tomadas de Cagigal (1979), de las características del juego de Huizinga (1968) y Caillois (1986), así como de algunos cursos desarrollados por la FIEP, como se planteó con anterioridad.

Coherente con el cambio de propuesta curricular en la formación de licenciados en educación física para 1984, los denominados juegos predeportivos adquieren preponderancia no solo como parte de las asignaturas denominadas “Teoría y Método de...", sino como actividades centrales en el desarrollo de las clases del área. Comparando la presencia del juego en el primer plan curricular con este, aquella se vio disminuida significativamente en su diversidad y aplicación, para centrarse en los juegos predeportivos, afirma MR5, ante lo cual de manera jocosa VCh4 comenta que "el pre-deportivo fue el rey del ochenta y cuatro".

SL7, quien realizó sus estudios de licenciatura en el marco de este currículo, plantea que en el desarrollo de las asignaturas denominadas “Teoría y Método..., el juego era utilizado como calentamiento o actividad para el inicio de la clase pero sin que sobre este hubiera trabajo teórico. La única intencionalidad que se podría interpretar de los docentes de estas asignaturas, era el uso de los denominados juegos predeportivos y, afirma SL7:

Yo vengo a ser consciente del juego como juego en las electivas y en el trabajo que hice para grado, ipero en las clases! Por eso te digo si los profesores nos ponían a jugar era lo que nosotros llamamos predeportivos. Pero ique nosotros hiciéramos un calentamiento con unos ponchados!, no, era directo al deporte y además como el aprendizaje se evaluaba con unas pruebas, entonces era directo a lo que tiene que hacer con el balón de baloncesto de fútbol o de voleibol.

Como estudiante, la reflexión sobre el juego, así como el acercamiento a autores como Huizinga (1968) y Caillois (1986) que lo trabajan, se dio por sugerencia de los asesores del proyecto de grado, los profesores Useche y Luis Alfonso Garzón, dice SL7:

[...] solo por allá en séptimo u octavo cuando ya comienzo a mirar lo que voy a hacer de trabajo de grado. Porque el trabajo de grado que nosotros hicimos con mi compañero de ese momento era proponer que a la piscina uno podía ir no solo a aprender a nadar, sino que uno podía ir a la piscina a jugar. Claro, eso ligado a una electiva que en ese momento se llamaba aqua-raquet y que era entrar a la piscina a jugar con raquetas. Nosotros le metimos coladores, nosotros le metimos tablas, nosotros le metimos un montón de cosas, para mostrar que sí, que uno puede hacer unos procesos de aprendizaje de la natación o más bien en ese momento de perderle miedo al agua porque era una cosa muy recurrente en nosotros, a través de juegos.

\section{EL TERCER PLAN CURRICULAR O PROYECTO CURRICULAR PARA LA LICENCIATURA EN EDUCACIÓN FÍSICA (PC-LEF)}

En este proyecto curricular iniciado en el año 2000, señala D06, la reflexión sobre el cuerpo se constituyó en su eje central y el juego no encontró allí un espacio explícito ni de amplia reflexión teórica. Ahora la denominación de las asignaturas evita la utilización de términos deportivos con la intención de superar la que en su momento se denominó deportivización de la educación física, minimizando la presencia de estas prácticas caracterizadas desde la institucionalidad federativa que las rige.

Por tanto, el deporte deja de ser un contenido explícito de enseñanza en la formación de los nuevos licenciados en educación física. Las llamadas asignaturas en los anteriores currículos, ahora se condensan en tres espacios académicos denominados desde el genérico "Formación Teórica" (Formación Teórica Humanística, Formación Teórica Pedagógica y Formación Teórica Disciplinar, con los Talleres de Cuerpo, de Lenguaje y de Experiencias Corporales), en los cuales es inviable la precisión de contenidos de enseñanza dada su generalidad temática. De esta manera, el juego, que en el primer plan curricular hacía presencia palmaria en la asignatura "Juegos Pedagógicos", vinculándose en otras asignaturas para los procesos de enseñanza/aprendizaje, y en el currículo del ochenta y cuatro se integraba en las asignaturas de deportes nombradas como "Teoría y Método de...", en la nueva estructura curricular no aparece de manera evidente.

Al ser considerado el juego como un recurso didáctico y no como un concepto estructurante de la educación física, es entendible que no tenga un desarrollo conceptual en el proyecto curricular PC-LEF, acota JJ1:

El asumirlo desde una tendencia didáctica y direccionado hacia incidir en los procesos de transformación del sujeto, que es un criterio novedoso para este currículo, no permite tomar de manera 
literal las clasificaciones del juego desarrolladas en el primer plan curricular, dado que esas formas de encasillamiento limitan la utilización de los juegos hacia el desarrollo humano.

Si la intencionalidad formativa es la que orienta las decisiones didácticas para la utilización del juego, entonces se comprende que los diferentes tipos o clasificaciones, posibilitan diversos aprendizajes y desarrollos, dependiendo de los núcleos temáticos de los espacios académicos que los asuman, lo cual exige del docente la creación de ambientes y condiciones de aprendizaje acordes con la finalidad prevista. Es claro que para esta dinámica planeación de los procesos educativos se hace necesario tener claridad sobre desarrollo de potenciales humanos y las particularidades o énfasis de los juegos para orientarlos en esa misma dirección.

Coherente con la perspectiva didáctica del juego, en el PC-LEF su estudio no se propone de manera explícita en espacios académicos o en alguno de los ciclos de formación de la licenciatura, sino que la comprensión y formación de los docentes son las que orientan los criterios para la aplicación del juego en los diferentes momentos de la formación de los licenciados, en correspondencia con las perspectivas fundamentales del proyecto curricular.

Con el ánimo de ejemplificar la nueva estructura curricular, anota JJ1:

El juego simbólico del que se había hablado antes aparece ahora como una estrategia de trabajo de representación corporal. En el tercer semestre de taller de cuerpo se tiene como núcleo temático el proceso representacional del cuerpo y se tienen todos aquellos juegos que tienen como esencia de acción la agrupación, la clasificación, la dramatización, son juegos representacionales.

De igual manera, comenta MR5, dentro del estudio de la temática "Desarrollo Motor", a partir de las características de las diferentes etapas, se proponen juegos coherentes con esos momentos evolutivos. Relacionado con lo anterior, afirma MR5, se desarrollan en el ciclo de fundamentación, talleres de rondas y de juegos para la infancia. Como producto de estas prácticas educativas se vienen elaborando cartillas de juegos para la educación física infantil, que a diferencia de las fichas del primer plan curricular, presentan desarrollos conceptuales, así como criterios para la enseñanza, con la intención de superar el instrumentalismo al que se ha tendido a reducir el juego en las respectivas clases.

En ese mismo ciclo, desde una perspectiva más general, SL7 considera coherente la presencia del juego como recurso para lograr diversas experiencias corporales y bien pueden ser juegos tradicionales, como juegos construidos con diversos elementos, entre estos los de deportes, sin que implique la práctica de técnicas específicas. Con la formación de ese amplio acervo de experiencias corporales, mediadas por diversidad de juegos, los estudiantes ingresan al segundo ciclo de formación y ese potencial les permite ahora acceder a aprendizajes de corte más técnico.

Explica JJ1

En décimo tenemos un espacio desde el taller de experiencias corporales, el juego como estrategia didáctica. Ahí se retoman cosas que habían sido trabajadas en los currículos anteriores para clasificar el juego, desde la perspectiva de uso, digamos así.

Y aquellas clasificaciones propuestas entran en vigencia, ahora atendiendo a la intencionalidad formativa de sujetos o subjetivación, propuesta para los procesos educativos. Para ello, llama la atención JJ1, es necesario comprender que las actividades propuestas para las respectivas clases y entre ellas el juego, no tienen sentido en sí mismas, lo cual llevaría a caer en el activismo o instrumentalización de los procesos, y eso es precisamente lo que se propone superar con el PC-LEF, a partir de tener siempre en consideración las intencionalidades formativas.

En esa misma línea argumentativa, SL7 aduce que la presencia explícita del juego en décimo semestre en la matriz de núcleos temáticos y temas derivados, obedece a la necesidad de fortalecer su comprensión para aplicarlo en las prácticas educativas que los estudiantes desarrollan en el trabajo de grado o Proyecto Curricular Particular (PCP), como en el cierre de su proceso de formación en la licenciatura. Ahora bien, ello implica que del primero al noveno semestre, el juego haya sido motivo de estudio en sus significados, características, clasificaciones y tipologías, así como desde perspectivas didácticas coherentes con aquellas, para superar la tendencia instrumentalista a la que históricamente se le tiende a reducir en las clases de educación física. 
A propósito de la denominada instrumentalización, AD8 la refiere como el simple hecho de enseñar juegos a los niños o entretenerlos jugando sin que exista una intención o significado, o bien reduciéndolos a, por ejemplo, mejorar las relaciones sociales o la trillada idea de hacer mejores personas. Es proponer juegos porque en el imaginario de la educación física estos deben estar presentes, pero sin un mínimo de raciocinio o análisis por parte del docente sobre los argumentos o criterios desde los cuales tomar las decisiones de instalarlos en las respectivas clases.

Afirma AD8: "Instrumentalización es no meterle cacumen a eso que va más allá de la actividad realizada", pues esta no se queda solamente en la acción sino que concomitante con ella se dan otros aspectos que influyen al alumno en algún sentido, pero sin que el docente se dé cuenta de ello y que bien puede estar dentro de lo requerido por la educación, como posiblemente en su contravía.

La importancia del juego, afirma JJ1, está además, en la creación de ambientes agradables y de disfrute para el aprendizaje, abriendo espacio a la manifestación de la dimensión lúdica, la cual puede comprenderse desde lo referido por Huizinga (1968) como espontaneidad y naturalidad.

Coincidente con estos planteamientos y en la línea de dar énfasis a lo formativo, HCh9 explica las alternativas que desde las prácticas deportivas viene desarrollando para superar el tecnicismo al que se han reducido en la educación física, en pos de los resultados. Ello se ha logrado vinculando las iniciales de inclusión, integración y equidad en la nominación de las nuevas prácticas, las cuales ahora se identifican, por ejemplo: voliboliie, softboliie o volipiie, etc., para desde el lenguaje darles nuevos sentidos y significados que se reflejan en la práctica.

Desde su experiencia docente, SL7 considera que las prácticas de juegos, sus innovaciones y la teorización correspondiente en el ciclo de fundamentación, permite a los estudiantes formar conceptos más claros sobre juego, deporte, recreación y actividad física, lo cual redunda en la clarificación de su futura misión docente. Para ello, continúa expresando, es oportuno que los grupos de docentes tengan claras estas necesidades y posibilidades de la formación profesional desde el juego, para que efectivamente se le dé espacio en el ciclo de fundamentación y a lo largo de la formación en la licenciatura, pues de no ser así los estudiantes no lograrían las suficientes comprensiones y aprendizajes en ese sentido.

A propósito, dice AD8:

Tuve la suerte de encontrarme con un grupo de profesores extraordinarios y tuve una experiencia muy bonita. Lo que vimos en un principio fue como este vértigo propio del juego, este desorden, sí la paidia diría ahora, en ese entonces no. Y entonces jugábamos, jugábamos mucho y perdíamos el control y de todo, desde un ponchados, hasta un ajedrez o un parqués, en clase como ejercicios de clase. Recuerdo mucho los salones de los espejos, hacíamos ejercicios corporales que no eran ni deporte ni danza ni ninguna práctica específica que conociéramos, pero metíamos de todo un poco y a eso lo llamábamos juego.

Pensando sobre la didáctica para la instalación del juego en el desarrollo de los diferentes espacios académicos, SL7 enfatiza en tomar el juego como medio para otros aprendizajes, es decir, el juego como mediación para enseñar algo. El juego como fin aún no se estudia suficientemente, sin embargo, plantea que las dos alternativas, ser medio o ser fin, pueden tener cabida en los procesos educativos pues así como en determinados momentos es utilizado para la enseñanza de contenidos externos al juego mismo, también se presentan otros en que se desarrolla por el juego mismo y la finalidad está en su propia realización y sin imponerle predeterminaciones.

En el diálogo con AD8 se coincide en la unidad dialéctica que en el juego se presenta entre ser fin y ser medio en los procesos educativos. Si bien es cierto, la esencia de uno se contrapone a la esencia del otro, ambos fenómenos se presuponen en una unidad condicionada en la cual, aunque con cierto énfasis en uno u otro en el desarrollo del juego, la posibilidad de aprender no coarta la expresión espontánea de los jugadores. Por el contrario, inmersos ellos en una realidad aparte de la cotidianidad, se entregan al juego por el juego mismo como expresión de su existencia, sin que la intención de aprender algo sea la única alternativa para su realización, y más bien los aprendizajes allí inherentes se constituyen en cualificación del hecho mismo de jugar.

La reflexión sobre el juego sigue siendo prioridad en la formación de los licenciados en educación física, de la cual es componente importante, afirma SL7. 
En ello coinciden los profesores y de manera sintética se pueden plantear para este propósito, campos de trabajo teórico como el desarrollo cognitivo, el antropológico, el filosófico, el pedagógico y didáctico, entre otros.

\section{CONCLUSIONES}

Sin duda el juego ha hecho presencia significativa en los diferentes planes curriculares de la formación de licenciados en educación física en la Universidad Pedagógica Nacional. Prueba de ello son los desarrollos teóricos y la diversidad de sus prácticas, que a lo largo de dicha formación profesional se han venido dando. Si bien es cierto, la tendencia ha sido hacia asumirlo desde perspectiva didáctica como mediación en los procesos educativos, también el reconocerlo como fin de dichos procesos viene teniendo cabida, principalmente desde la reflexión particular de algunos docentes, aunque sin el suficiente arraigo institucional o programático.

A partir de las perspectivas que sobre el juego se vienen dando en la actualidad, desde diferentes disciplinas como la filosofía, la antropología, la pedagogía y la didáctica, principalmente, es pertinente revisar tanto las prácticas como los desarrollos teóricos que en esta formación de licenciados en educación física se ha venido presentando sobre el juego y sus aportes a la formación del potencial humano. Continuar pensando el juego en esta línea de trabajo aspira a superar definitivamente la tendencia instrumentalista que ha caracterizado la presencia del juego como actividad didáctica en las clases de educación física.

La comprensión del juego en el ámbito de la educación física, ya no solamente como mediación sino también como fin en los procesos de enseñanza/aprendizaje, exige continua reflexión y diálogo académico de los docentes para lograr la suficiente clarificación que permita su instalación en el medio didáctico, de manera coherente con esa complejidad que lo caracteriza. "La apertura lúdica de la existencia humana hacia el fundamento lúdico del ser", planteada por Fink (1966, p. 29); en este mismo sentido Holzapfel (2003) al afirmar que "hay, pues, una dimensión lúdica de la existencia humana" (p. 71); así como "el juego es la condición de posibilidad de lo lúdico como ejercicio de la libertad y de la creatividad humana" (MEN, 2010), se constituyen en horizonte de trabajo para esta línea de pensamiento.
Los desarrollos conceptuales y la amplia utilización del juego en los procesos formativos de la educación física, tanto en la formación profesional, como su proyección a la educación básica y media, a la educación para el trabajo y el desarrollo humano y a la educación informal, exigen de mayor sistematicidad a lo largo de los ciclos de formación de los profesionales y de los diferentes semestres, para fundamentarlo en el ámbito formativo de la educación física, de tal manera que se conjuguen sus dos grandes perspectivas de ser medio y ser fin para el desarrollo del potencial humano.

El debate sobre el juego y el deporte en el contexto de la educación física, es de total vigencia en la actualidad. Inquietudes sobre si hay presencia del juego en las prácticas deportivas aún no están resueltas y la superación de aquellas no puede reducirse a la exclusión de algunas prácticas sin la suficiente autocrítica y reflexión. Muy oportunas las apreciaciones de AM10 al respecto. Si bien son claras sus diferencias entre el juego y el deporte, sus palabras muestran un interesante horizonte para el deporte en la educación, a partir de llevarlo al nivel del arte.

No puede desaparecer jamás, es un logro de nuestra especie, es un refinamiento, pero nosotros lo llevamos a un extremo nefasto. Una salida grande es hacer del deporte un arte y entonces, cuando uno convierte las cosas que hace en arte es cuando eso fluye, no hay esfuerzo, no me importa en últimas el resultado. Y eso va como tan adentro de mí que esa es la forma en que yo lo sé vivir y fluyo dentro de ello y no me cuesta trabajo, no me estresa. $\mathrm{Y}$ eso es como que ya entrenó ocho años siete horas y ahora me siento autónomo y hasta con los ojos cerrados y salen cosas bonitas y entonces el deporte uno también podría llevarlo a unos niveles tales de fluidez, de ausencia de esfuerzo y eso es un logro espiritual es una conquista interna y eso no es fácil. Esa es la diferencia entre no conocer, entendido como ignorancia, a entretener el no conocer entendido como aquel que se olvida de todas las cosas que ha aprendido en la vida, porque ya logró introducirlo dentro de sí de tal manera que ni siquiera lo explica mediante palabras, sino que vive absolutamente ausente de cualquier conflicto y de contradicción. Eso es fregao, pero yo lo veo por ahí, que uno puede hacer del deporte un completo arte.

En este mismo sentido, es necesario volver a estudiar a Caillois (1986) y la clasificación de juegos que propone, para re-significarla en el ámbito de la educación física. 
La tendencia que en las prácticas educativas de esta área de la educación se ha dado hacia los juegos agon, entre los cuales se vincula al fútbol (Caillois, 1986, p. 41), puede reconsiderarse para dar espacio a las otras categorías (ilinx, mimicry, alea). Es el caso de los denominados deportes extremos que gran auge han alcanzado en la actualidad, con significativa presencia en los diversos ámbitos educativos.

Para la construcción del conocimiento profesional del profesor de educación física es fundamental dar continuidad a la reflexión sobre el juego y las transposiciones didácticas necesarias para constituirlo en conocimiento disciplinar del área. Algunos ejemplos pueden ser orientadores, como el paso de la inteligencia práctica a la representativa y para lo cual el juego se puede considerar como zona de desarrollo próximo (Vygotsky, 1978). 0 el desarrollo del sentido de las reglas, que desde Piaget propone Seybold (1976) para la educación física, en reglas motrices, reglas coercitivas de observancia unilateral y reglas racionales de observancia mutua. Así mismo, el potencial pedagógico del juego a partir de su condición dialógica, la cual solo es posible en la existencia humana como corporeidad (Gruppe, 1976). En el campo de la enseñanza es importante la revisión del juego como modelo didáctico, según propone Amade-Escot (2009), para el diseño medios didácticos en los que sea este la estrategia para la confrontación del estudiante con los contenidos a aprender. Para ello el profesor se integra al juego en el cual no todo está predeterminado, sino que se va construyendo colectivamente (profesor-estudiantes), desde lo que la autora denomina ingenio didáctico.

Las diferentes clasificaciones propuestas, la diversidad de juegos construidos o involucrados en las prácticas educativas, las teorías revisadas, los lineamientos pedagógicos y didácticos trazados constituyen ya un corpus de conocimiento importante para la educación física, desde el cual continuar en esa estructuración conceptual y argumentativa.

Definitivamente es necesario seguir pensando el juego en el ámbito de la educación física.

\section{REFERENCIAS}

Amade-Escot, C. (2009). La didáctica de la educación física deporte de alto nivel. Buenos Aires: Stadium.

Cagigal, J. (1979). Cultura intelectual y cultura física. Buenos Aires: Kapelusz.

Caillois, R. (1967). Les jeux et les Hommes. Le masque et le vertige. París: Editions Gallimard.

Caillois , R. (1986). Los juegos y los hombres. México: Fondo de Cultura Económica S. A.

De la Ferriére, S. (s.f.). Yug yoga yoguismo. Recuperado de http://www.sergeraynauddelaferriere.net/obras/ yyy/01/yyy01-introduccion.pdf

Fink, E. (1966). Oasis de la felicidad. Trad. E. Frost. México: UNAM.

Gruppe, 0. (1976). Teoría pedagógica de la educación física. Trad. D. Romero. Madrid: INEF.

Holzapfel, C. (2003). Crítica de la razón lúdica. Madrid: Trotta, S. A.

Huizinga, J. (1968). Homo Ludens. Buenos Aires: Emecé Editores, S. A.

Meinel, K. (1977). Didáctica del movimiento. Trad. J. Vilar. La Habana: Orbe.

Ministerio de Educación Nacional (MEN) (2010). Orientaciones Pedagógicas para la Educación Física, Recreación y Deporte. Bogotá D. C., Colombia.

Morin, E. (1999). Los siete saberes necesarios para la educación del futuro. Trad. M. Vallejo-Gómez. París: Unesco.

Mosston, M. (1978). La enseñanza de la educación física del comando al descubrimiento. Buenos Aires: Paidós.

Ortega, J. (s.f.). Julio Cortázar entre todos los juegos. Recuperado de www.revistadelauniversidad.unam. $\mathrm{mx} / 0104 /$ pdfs/julio_cortazar.pdf

Paredes, J. (2003). Juego, luego soy teoría de la actividad lúdica. Sevilla: Wanceulen Editorial Deportiva, S. L.

Seybold, A. (1976). Principios didácticos de la educación física. Buenos Aires: Kapelusz.

Vaca, A. (1998). Historia del alma mater de la Educación Física en Colombia. Santa Fe de Bogotá: ARFO Ltda.

Vygotsky, L. (1978). El desarrollo de los procesos psicológicos superiores. Barcelona: Grijalbo. 


\section{DIÁLOGOS}

Judit Jaramillo (JJ1). Egresada de la Escuela Nacional de Educación Física y profesora en los tres planes curriculares de educación física en la Universidad Pedagógica Nacional. Coautora del actual currículo denominado PC-LEF.

Fernando Uribe (FU2). Egresado del primer plan curricular de Educación Física en la Universidad Pedagógica Nacional y profesor en los tres currículos.

Clara Peña (CP3). Egresada del primer plan curricular de Educación Física en la Universidad Pedagógica Nacional y profesora en los tres currículos.

Víctor Chinchilla (VCh4). Egresado del primer plan curricular de Educación Física en la Universidad Pedagógica Nacional y profesor en los tres currículos.

Martha Reyes (MR5). Egresada del primer plan curricular de Educación Física en la Universidad Pedagógica Nacional y profesora en los tres currículos.
Daniel Oliveros (D06). Egresado del primer plan curricular de Educación Física en la Universidad Pedagógica Nacional y profesor en los tres currículos.

Sonia López (SL7). Egresada del segundo plan curricular de Educación Física en la Universidad Pedagógica Nacional y profesora en los últimos dos currículos.

Andrés Díaz (AD8). Egresado del tercer plan curricular de Educación Física en la Universidad Pedagógica Nacional y profesor en la Licenciatura en Recreación y Turismo.

Hernando Chávez (HCh9). Egresado del primer plan curricular de Educación Física en la Universidad Pedagógica Nacional y profesor en los tres currículos.

Alfonso Martín (AM10). Egresado del primer plan curricular de Educación Física en la Universidad Pedagógica Nacional y profesor en los tres currículos. 